\title{
Noninvasive ventilation for prevention of post-extubation respiratory failure in obese patients
}

\author{
A.A. El Solh*, A. Aquilina*, L. Pineda*, V. Dhanvantri*, B. Grant*** and P. Bouquin*
}

ABSTRACT: Current recommendations for management of obese patients post-extubation are based on clinical experience and expert opinions. It was hypothesised that the application of noninvasive ventilation (NIV) during the first $48 \mathrm{~h}$ after extubation in severely obese patients would reduce post-extubation failure and avert the need for reintubation.

Following protocol-driven weaning trials, 62 consecutive severely obese patients (body mass index $\geqslant 35 \mathrm{~kg} \cdot \mathrm{m}^{-2}$ ) were assigned to NIV via nasal mask immediately post-extubation and compared with 62 historically matched controls who were treated with conventional therapy. The primary end-point was the incidence of respiratory failure in the first $\mathbf{4 8} \mathbf{h}$ post-extubation.

Compared with conventional therapy, the institution of NIV resulted in $16 \%$ (95\% confidence interval 2.9-29.3\%) absolute risk reduction in the rate of respiratory failure. There was a significant difference in the intensive care unit and lengths of hospital stay between the two groups. Subgroup analysis of hypercapnic patients showed reduced hospital mortality in the NIV group compared with the control group.

In conclusion, noninvasive ventilation may be effective in averting respiratory failure in severely obese patients when applied during the first $48 \mathrm{~h}$ post-extubation. In selected patients with chronic hypercarbia, early application of noninvasive ventilation may confer a survival benefit.

\section{KEYWORDS: Noninvasive ventilation, obesity, obstructive sleep apnoea, reintubation}

$\mathbf{N}$ umerous studies have highlighted the complexity of respiratory management of critically ill obese patients during the period following liberation from mechanical ventilation $[1,2]$. The development of respiratory instability, episodic desaturation during supine position, and the respiratory depressant effects of sedatives and opioid analgesia predispose these patients to prolonged periods of apnoeas, hypoxia and severe hypercapnia culminating in respiratory failure. Yet the incidence of these serious complications is not well documented. The rate of reintubation post-extubation in severely obese patients has been reported at 8$14 \%$ among patients undergoing mechanical ventilation for $>48 \mathrm{~h}[3,4]$, but these estimates are likely to underestimate the true incidence of respiratory failure in this population.

Noninvasive ventilation (NIV) has been considered a promising therapy by an International Consensus Conference to avert respiratory failure after weaning [5]. However, randomised controlled studies in nonobese patients have shown mixed results. While earlier studies failed to show any benefit from rescue NIV [6, 7], a more recent investigation has documented a beneficial effect of administering NIV immediately postextubation in selected patients at increased risk for extubation failure [8]. FERRER et al. [8] have shown not only a reduction in the rate of respiratory failure but also a decrease in the rate of intensive care unit (ICU) mortality. As severely obese patients are considered at high risk of developing respiratory complications [1, 9], the current authors instituted a protocol requiring early application of NIV post-extubation. It was hypothesised that the preventive use of NIV would reduce the incidence of respiratory failure and the rate of reintubation. To test this hypothesis, an observational study was conducted comparing NIV therapy to historical controls of conventional medical therapy during the first $48 \mathrm{~h}$ post-extubation.

\section{METHODS}

\section{Patient population}

In a prospective study beginning in September 2004, involving 52 beds in three ICUs, all severely obese patients (body mass index (BMI) $\geqslant 35$ $\mathrm{kg} \cdot \mathrm{m}^{-2}$ ) requiring endotracheal mechanical ventilation for at least $48 \mathrm{~h}$ at a university-affiliated

\section{AFFILIATIONS}

*Western New York Respiratory Research Center, Depts of Medicine and of Biostatistics, Division of Pulmonary, Critical Care, and Sleep Medicine, University at Buffalo, and \#Veterans Affairs Medical Center, Buffalo, NY, USA.

CORRESPONDENCE

A.A. El Solh

Division of Pulmonary

Critical Care

and Sleep Medicine

Erie County Medical Center

462 Grider Street

Buffalo

NY 14215

USA

Fax: 17168986139

E-mail: solh@buffalo.edu

Received:

December 212005

Accepted after revision:

May 112006

\section{SUPPORT STATEMENT}

None of the authors have a financial relationship with a commercial entity that has interest in the subject of this manuscript. This study was

supported by a grant from Research for Health in Erie County, NY, USA.

European Respiratory Journal Print ISSN 0903-1936

Online ISSN 1399-3003 
tertiary care centre were eligible for enrolment. Exclusion criteria included recent facial, cranial or cervical spine surgery, neuromuscular disorders, uncontrolled delirium, active upper gastrointestinal bleeding and unplanned extubation. Patients who had a do-not-resuscitate order were also excluded. For those patients with more than one ICU admission, only the first event was included in the analysis to ensure independence of observation. A historic control group of patients who met the same inclusion and exclusion criteria was chosen from severely obese patients admitted to the ICU in the preceding 29 months. The NIV and the historic group were matched for age $( \pm 5 \mathrm{yrs}), \mathrm{BMI}\left( \pm 2 \mathrm{~kg} \cdot \mathrm{m}^{-2}\right)$, and Acute Physiology and Chronic Health Evaluation (APACHE II) score $( \pm 2)$ [10]. For each patient in the NIV group, matching was performed with a patient from the historic group according to the following hierarchy: baseline APACHE II score, BMI and age. When matching was unsuccessful at end of this process, it was started again with extension of the age for matching ( $\pm 5 \mathrm{yrs})$ and then extension of the range of BMI $\left( \pm 2 \mathrm{~kg} \cdot \mathrm{m}^{-2}\right)$. If there was more than one match, the patient in the historic group was selected by the best match according to the matching hierarchy. Using this algorithm, matches for all the NIV patients were identified.

Study approval was obtained from the local Institutional Review Board prior to initiation of the study. Written informed consent was waived owing to the observational nature of the study.

\section{Weaning protocol}

Starting in February 2002, weaning trials were conducted according to a previously established protocol [11]. In brief, a weaning trial was initiated once the patient was fully awake, responsive, and had fulfilled the following criteria: arterial oxygen tension $\left(\mathrm{Pa}_{\mathrm{a}} \mathrm{O}_{2}\right)>9.31 \mathrm{kPa}$, with inspiratory oxygen fraction $\left(\mathrm{FI}, \mathrm{O}_{2}\right) \leqslant 0.35$ and positive end-expiratory pressure $\leqslant 5 \mathrm{cmH}_{2} \mathrm{O}$; rectal temperature $>36.6^{\circ} \mathrm{C}$; a stable cardiovascular system (i.e. systolic blood pressure $>100 \mathrm{mmHg}$, heart rate $<120$ beats $\cdot \mathrm{min}^{-1}$ ); and absence of metabolic acidosis and withdrawal of all vasopressors for $>12 \mathrm{~h}$. The screen for readiness to wean was performed between 07:00 $\mathrm{h}$ and 09:00 $\mathrm{h}$ based on a previously published weaning protocol shown to reduce duration of ventilation [11]. During screening, the ratio of respiratory frequency to tidal volume $(f \mathrm{R} / V \mathrm{~T})$ was assessed [12]. If $f R / V T$ was $<105 \mathrm{~min}^{-1} \cdot \mathrm{L}^{-1}$, a spontaneous breathing trial was attempted for $30 \mathrm{~min}$ on continuous positive airway pressure (CPAP), with $5 \mathrm{cmH}_{2} \mathrm{O}$ pressure support if the endotracheal tube was $\leqslant 8 \mathrm{~mm}$. Extubation was then ordered by the attending physician, otherwise the patient was placed back on mechanical ventilation if within $30 \mathrm{~min}$ of initiating spontaneous breathing trial (SBT) any of the following criteria were met: 1) change in heart rate of $>20$ beats $\cdot \mathrm{min}^{-1}$ from baseline prior to initiating SBT persisting for $\geqslant 5 \mathrm{~min} ; 2$ ) systolic blood pressure of $<90 \mathrm{mmHg}$ or change of $>30 \mathrm{mmHg}$ after initiating SBT persisting for $\geqslant 5 \mathrm{~min}$; 3) arterial oxygen saturation $\left(\mathrm{Sa}_{\mathrm{a}} \mathrm{O}_{2}\right)<90 \%, \mathrm{~Pa}_{2} \mathrm{O}_{2}<7.98 \mathrm{kPa}$, or $\mathrm{pH}$ $\leqslant 7.35$; and 4) respiratory muscle fatigue or increased work of breathing suggested by the use of accessory respiratory muscles, paradoxical motion of the abdomen, or retraction of the intercostal spaces.
Post-extubation, patients were placed on conventional medical therapy, including oxygen therapy to maintain $\mathrm{Sa}_{2} \mathrm{O}_{2}$ at $>90 \%$, bronchodilators, chest physiotherapy and other treatments directed by the primary-care physicians.

\section{Noninvasive ventilation}

In July 2004, the current authors adopted a protocol in ICUs to administer NIV to severely obese patients for the first $48 \mathrm{~h}$ immediately post-extubation. NIV was delivered using the bilevel positive airway pressure mode (BiPAP S/T-D Ventilatory Support System; Respironics Inc., Murrysville, PA, USA) via a nasal mask. Nasogastric tubes were removed prior to institution of NIV. The inspiratory and expiratory pressures were initially set at $12 \mathrm{cmH}_{2} \mathrm{O}$ and $4 \mathrm{cmH}_{2} \mathrm{O}$, respectively, in a spontaneous mode [13]. The pressure settings were increased gradually to the patient's tolerance with the aim of achieving a respiratory rate $<25$ breaths $\cdot \mathrm{min}^{-1}$ and $\mathrm{Sa}_{2} \mathrm{O}_{2}$ $>90 \%$. The fractional concentration of oxygen was also titrated to maintain an $\mathrm{Sa}_{1} \mathrm{O}_{2}>90 \%$. Subjects were advised to use NIV continuously with periods of rest up to 60 min every $6 \mathrm{~h}$ to receive meals, scheduled medications or nursing care. Regular examination of the facial skin was made by the respiratory therapist to prevent skin ulceration from the tight-fitting mask. NIV was maintained for the first $48 \mathrm{~h}$, then the decision to maintain or discontinue NIV was left to the admitting physician.

Medical care was provided by the same intensivist team, critical care nurses and respiratory therapists during the study period.

\section{Criteria for respiratory failure and reintubation}

Reintubation was performed immediately post-extubation when any of the following major clinical events occurred: respiratory or cardiac arrest; irregular respiratory rate associated with loss of consciousness or gasping for air; and severe haemodynamic instability without response to fluids and vasoactive drugs [14]. In addition, respiratory failure was defined as the presence or persistence after $1 \mathrm{~h}$ post-extubation of any of the following parameters requiring reintubation or rescue NIV: 1) hypercapnia (arterial $\mathrm{pH}<7.35$ along with an increase in carbon dioxide arterial tension $\left(\mathrm{Pa}_{\mathrm{a}} \mathrm{CO}_{2}\right)$ of $>20 \%$ from the time of extubation); 2) hypoxaemia defined as $\mathrm{Sa}_{2} \mathrm{O}_{2}$ by pulse oximetry $<90 \%$ or $\mathrm{Pa}_{1} \mathrm{O}_{2}<7.98 \mathrm{kPa}$ with $\mathrm{FI}_{1} \mathrm{O}_{2}>0.5$; 3) decreased consciousness or psychomotor agitation rendering the patient unable to tolerate NIV; 4) clinical signs suggestive of respiratory muscle fatigue and/or increased work of breathing, such as the use of respiratory accessory muscles, intercostal indrawing, or paradoxical motion of the abdomen; 5) severe delirium or agitation; and 6) inability to clear secretions.

\section{Data collection}

Sociodemographic and clinical data were analysed. These included age, sex, BMI, reason for mechanical ventilation, APACHE II score on admission to the ICU and at the time of liberation from mechanical ventilation, and duration of mechanical ventilation before extubation. Respiratory rate, heart rate, spontaneous tidal volume and the rapid shallow breathing index were obtained prior to extubation. The causes for respiratory failure were classified as described in the 
preceding section. In the historic group, the need for rescue NIV in the first $48 \mathrm{~h}$ post-extubation was considered a failure of the standard medical therapy. Complications recorded postextubation included hospital-acquired pneumonia and nosocomial bloodstream infection irrespective of its origin. These were defined according to the Centers for Disease Control criteria [15]. At the time of discharge, the total lengths of stay and hospital mortality rate were recorded.

\section{Statistical analysis}

The primary end-point was the incidence of respiratory failure in the first $48 \mathrm{~h}$ post-extubation. After reviewing reintubation rates in critical care units in this high-risk group over a $1 \mathrm{yr}$ period, 62 patients were required in each group in order to detect a $20 \%$ absolute reduction in the risk of respiratory failure with the application of prophylactic NIV relative to $25.8 \%$ in the conventionally treated group, with a power of $80 \%$ and a type I error of $5 \%$. Secondary outcomes were lengths of ICU and hospital stay and hospital mortality.

Results are expressed as mean $\pm \mathrm{SD}$. Continuous variables for the two groups were compared with an unpaired t-test for normally distributed data or the Mann-Whitney U-test otherwise. Qualitative or categorical variables were compared with the Chi-squared test or Fisher's exact test. A difference was considered statistically significant when the alpha probability was $<0.05$ (all two-tailed).

\section{RESULTS}

Over the period from February 2002 to June 2004, 101 severely obese patients who required endotracheal intubation for $\geqslant 48 \mathrm{~h}$ were identified. Eighteen had tracheostomy placed without extubation trial, one was transferred to another facility, and two had a terminal weaning. Eighty eligible patients were then considered for inclusion. Seven patients were excluded for the following reasons: uncontrolled delirium $(n=3)$, unplanned extubation $(n=2)$, facial surgery $(n=1)$, and cranial surgery $(n=1)$, leaving 73 patients in a pool to access for matching to the NIV group. From July 2004 to September 2005, 83 severely obese patients required intubation $\geqslant 48 \mathrm{~h}$. Fourteen patients had a tracheostomy and one had a terminal weaning. Six patients met one of the exclusion criteria: unplanned extubation $(n=3)$, do-not-resuscitate order $(n=1)$, uncontrolled delirium $(n=1)$, and facial surgery $(n=1)$. The remaining 62 patients of the NIV group were then matched to the conventionally treated participants (fig. 1). The two groups were similar in age, sex, BMI, underlying comorbidities and APACHE II score (table 1). Similarly, the causes for endotracheal mechanical ventilation were comparable in both groups. Obstructive apnoea was documented in $22 \%$ of the NIV group and $27 \%$ of the conventional medical therapy group. The corresponding mean $\pm \mathrm{SD}$ apnoea-hypopnoea indices were $45.4 \pm 7.5$ events $\cdot \mathrm{hr}^{-1}$ (range 14-112) and $40.8 \pm 6.3$ events $\cdot \mathrm{hr}^{-1}$ (range 6-94; $p=0.64$ ). None of the patients in the historic group had CPAP therapy in the first $48 \mathrm{~h}$ post-extubation.

Table 2 displays the physiological indices of participants prior to extubation. In the NIV group, the mean \pm SD inspiratory and expiratory positive airway pressures were $17.2 \pm 3.5 \mathrm{cmH}_{2} \mathrm{O}$ (range 12-26) and $7.2 \pm 1.6 \mathrm{cmH}_{2} \mathrm{O}$ (range 5-12), respectively. The mean \pm SD use of NIV was $16.2 \pm 2.6 \mathrm{~h} \cdot$ day $^{-1}$ for the first $48 \mathrm{~h}$ post-extubation. Nine patients complained of oral dryness due to an inability to keep a tight seal. Four patients responded to placement of a chinstrap and five were switched to a full face mask. Two patients had abrasion of the dorsum nasae but none of the patients developed evidence of gastric distension, aspiration or epistaxis. Twenty-three patients remained on the bilevel positive pressure ventilation beyond the $48 \mathrm{~h}$; of these, 17 showed evidence of hypercapnia during a trial of spontaneous breathing. In comparison, the mean \pm SD inspiratory and expiratory positive airway pressures for the conventionally treated group were $15.5 \pm 3.5 \mathrm{cmH}_{2} \mathrm{O}$ (range 10-22) and $6.4 \pm 2.2 \mathrm{cmH}_{2} \mathrm{O}$ (range 4-10), respectively, but the difference was not statistically different between the two groups $(p=0.17$ for inspiratory positive airway pressures and $\mathrm{p}=0.2$ for expiratory positive airway pressures, respectively). In contrast, the average use of NIV was significantly lower at $9.8 \pm 5.8 \mathrm{~h}(\mathrm{p}<0.001)$ as eight of the 12 severely obese patients who received rescue NIV required reintubation within the first $24 \mathrm{~h}$ after the onset of respiratory failure.

The institution of NIV post-extubation resulted in a $16 \%(95 \%$ confidence interval (CI) $2.9-29.3 \%$ ) absolute reduction in the risk of respiratory failure compared with the conventionally treated group (10 versus 26\%; relative risk (RR) 0.38 ; $95 \% \mathrm{CI}$ $0.16-0.89)$. Table 3 outlines the causes of respiratory failure in both groups. As NIV rescue therapy resulted in three patients avoiding reintubation out of the 12 receiving standard therapy, the rate of reintubation was not statistically significant between the two groups (10 versus 21\%; RR 0.46; 95\% CI 0.19-1.14). The majority of events responsible for respiratory failure occurred in the first $24 \mathrm{~h}$ as 19 out of the 22 cases of respiratory failure were reported $8.6 \pm 4.9 \mathrm{~h}$ post-extubation. The time to respiratory failure post-extubation was longer for the NIV group (16.0 $\pm 8.9 \mathrm{~h}$ ) relative to those assigned to the standard therapy $(8.6 \pm 6.1 \mathrm{~h})$ but the difference fell short of statistical significance $(\mathrm{p}=0.14)$.

Although the rate of nosocomial bloodstream and respiratory infections was not significantly different between the two groups, patients who received NIV therapy had shorter ICU and hospital lengths of stay compared with those who were assigned to the conventional medical therapy $(p<0.001$ and $p=0.007$, respectively). Hospital mortality, however, was comparable between the NIV group and the conventional therapy group.

In a post hoc analysis of the 47 patients who had hypercapnia during a trial of spontaneous breathing, the incidence of respiratory failure was significantly reduced in those assigned to the NIV group $(p=0.03$; table 4$)$. There was also a trend towards a reduction in the frequency of reintubation $(p=0.1)$. Bloodstream and respiratory infections were observed in four $(16 \%)$ out of 25 in the NIV group with hypercapnia and 12 $(55 \%)$ out of 22 in the conventional therapy group $(p=0.01)$. As a result, the mortality rate was significantly higher for patients with hypercapnia assigned to conventional therapy $(p=0.03)$. Respiratory failure with prolonged mechanical ventilation was responsible for eight out of the 11 deaths in the conventional therapy group, compared with two out of the four deaths in the hypercapnic NIV group $(p=0.56)$.

\section{DISCUSSION}

The main finding of the present study is that the use of NIV therapy in severely obese patients resulted in a $16 \%$ absolute 


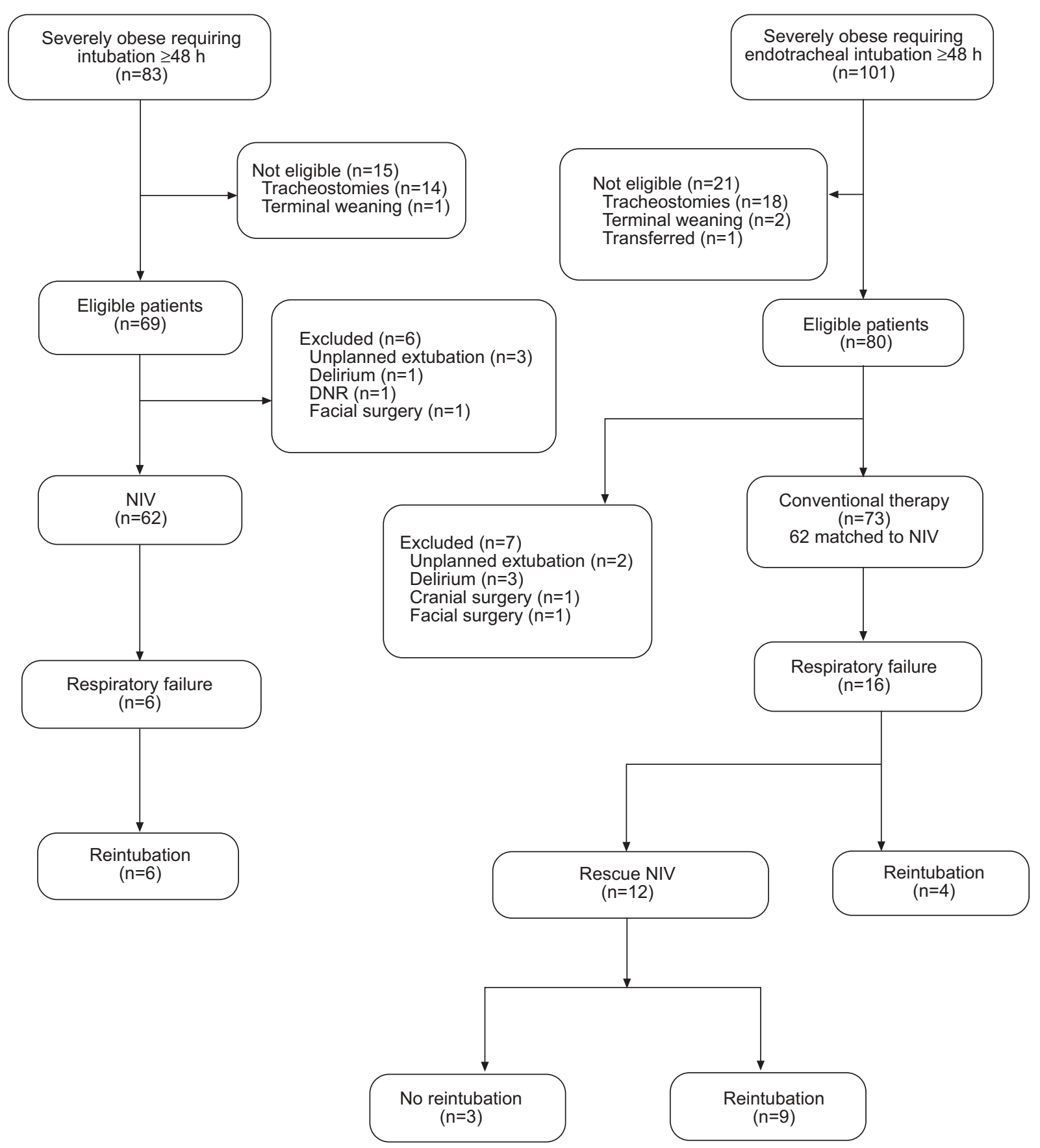

FIGURE 1. Flow diagram of the study population. DNR: do not resuscitate; NIV: noninvasive ventilation.

reduction in the rate of respiratory failure post-extubation compared with conventional medical therapy. NIV was notably more beneficial in those who showed evidence of persistent hypercapnia during weaning trials.

Earlier investigations suggested that the prophylactic use of NIV in morbidly obese patients during the first $24 \mathrm{~h}$ postoperatively significantly reduced pulmonary dysfunction after gastroplasty and accelerated re-establishment of pre-operative pulmonary function [13]. JORIs et al. [13] demonstrated that the application of bilevel positive airway pressure set at $12 \mathrm{cmH}_{2} \mathrm{O}$ and $4 \mathrm{cmH}_{2} \mathrm{O}$ significantly improved peak expiratory flow rate, forced vital capacity and $\mathrm{Sa}_{1} \mathrm{O}_{2}$ on the first post-operative day. This improvement was attributed to a combined effect of improved lung inflation, prevention of alveolar collapse and reduced inspiratory threshold load. However, the study did not investigate the potential benefit of bilevel positive airway pressure on the incidence of post-operative pulmonary complications nor did it assess the impact on mortality or length of stay.

Subsequent studies that have examined the role of NIV in the post-extubation period have assessed outcomes only after respiratory failure has occurred. Two randomised controlled 
TABLE 1 Baseline characteristics of the study population

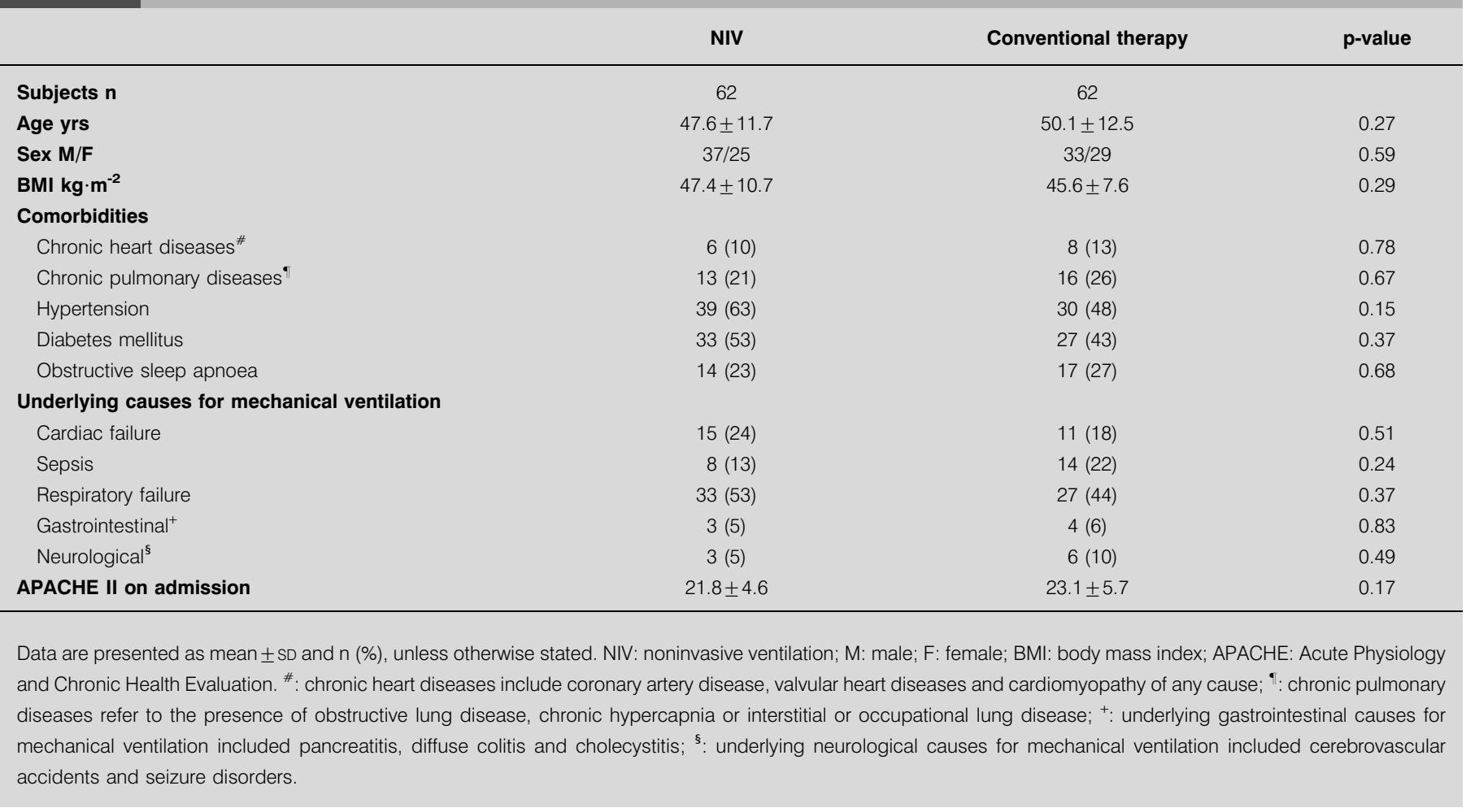

trials [6, 7] involving a heterogenous group of patients found that the use of NIV post-extubation did not reduce the rate of reintubation when compared with standard medical therapy. In contrast, the current study found that early intervention with NIV post-extubation reduced the frequency of respiratory failure and demonstrated a trend toward avoiding reintubation. The difference in these observations can be explained by the fact that the current protocol required immediate institution of NIV post-extubation rather than intervention after clinical signs of respiratory failure became evident. Once respiratory distress ensues, delay in reversing respiratory failure has been shown to contribute to organ dysfunction and poor outcomes [16]. Along this line, JIANG et al. [17] examined the efficacy of prophylactic NIV in a randomised trial by assigning 93 patients to either biphasic positive airway pressure or unassisted oxygen therapy, but the difference in rates of reintubation in the trial was not statistically significant between the two groups. However, the study recruited participants "indiscriminately", including those with unplanned extubation. The relevance of patient selection was highlighted in two recently published trials [18, 19]. In a multicentre randomised controlled trial, NAVA et al. [18] found

\section{TABLE 2 Characteristics of the patients at the time of extubation}

\begin{tabular}{|c|c|c|c|}
\hline Subjects $n$ & 62 & 62 & \\
\hline Duration of mechanical ventilation days & $7.9 \pm 3.6$ & $8.8 \pm 4.2$ & 0.23 \\
\hline \multicolumn{4}{|l|}{$\begin{array}{l}\text { Physiological indices during } \\
\text { spontaneous breathing trial }\end{array}$} \\
\hline Heart rate $\mathrm{min}^{-1}$ & $96.9 \pm 18.8$ & $99.3 \pm 19.9$ & 0.51 \\
\hline $\mathrm{fR} / \mathrm{V}$ T ratio $\mathrm{min}^{-1} \cdot \mathrm{L}^{-1}$ & $74.6 \pm 11.6$ & $71.5 \pm 13.7$ & 0.16 \\
\hline Arterial $\mathrm{pH}$ & $7.39 \pm 0.05$ & $7.4 \pm 0.06$ & 0.58 \\
\hline $\mathrm{Pa}, \mathrm{CO}_{2} \mathrm{mmHg}$ & $47.1 \pm 8.9$ & $44.8 \pm 7.7$ & 0.13 \\
\hline $\mathrm{Pa}_{\mathrm{a}_{2}} \mathrm{mmHg}$ & $83 \pm 14$ & $84 \pm 12$ & 0.67 \\
\hline $\mathrm{Pa}_{1} \mathrm{O}_{2} / \mathrm{Fl}, \mathrm{O}_{2}$ & $215 \pm 38$ & $221 \pm 41$ & 0.38 \\
\hline
\end{tabular}

Data are presented as mean $\pm \mathrm{SD}$, unless otherwise stated. NIV: noninvasive ventilation; APACHE: Acute Physiology and Chronic Health Evaluation; $\mathrm{fR} / \mathrm{VT}$ : ratio of respiratory frequency to tidal volume; $\mathrm{Pa}_{1} \mathrm{CO}_{2}$ : carbon dioxide arterial tension; $\mathrm{Pa}_{1} \mathrm{O}_{2}$ : oxygen arterial tension; $\mathrm{Fl}_{1} \mathrm{O}_{2}$ : inspiratory oxygen fraction. $1 \mathrm{mmHg}=0.133 \mathrm{kPa}$. 
TABLE 3 Post-extubation complications of critically ill morbidly obese patients

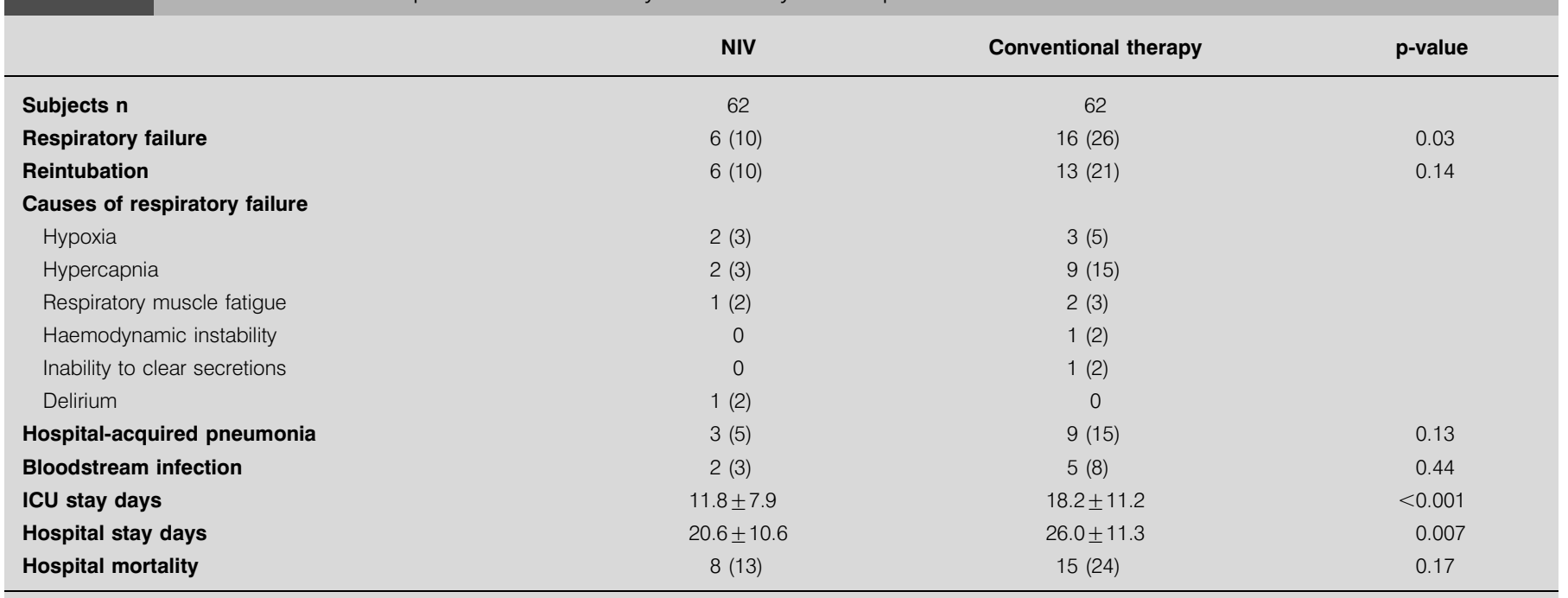

Data are presented as $n(\%)$ and mean $\pm S D$, unless otherwise stated. NIV: noninvasive ventilation; ICU: intensive care unit.

prophylactic NIV to be more effective in preventing reintubation than standard medical therapy in patients at risk. FERRER et al. [19] reached a similar conclusion by showing that the early use of NIV averted respiratory failure in selected patients considered at risk for respiratory failure post-extubation.

Another potential explanation for the observed benefit in the NIV cohort pertains to the fact that this study population is considered at high risk of obstructive sleep apnoea (OSA). Although the authors were aware of the presence of OSA in $25 \%$ of the study population, unrecognised OSA has been implicated in worsening respiratory failure and unexpected transfer to the ICU after liberation from mechanical ventilation [20, 21]. GUPTA et al. [22] reported $24 \%$ serious complications post-hip and -knee replacement in 101 patients with a diagnosis of OSA within $72 \mathrm{~h}$ post-extubation including reintubation and urgent CPAP. In the absence of high-quality evidence supporting the routine use of CPAP in severely obese patients with OSA after liberation from mechanical ventilation, the early application of NIV in the current study might have

\begin{tabular}{|c|c|c|c|}
\hline \multirow[t]{2}{*}{ TABLE 4} & \multicolumn{3}{|c|}{$\begin{array}{l}\text { Characteristics of patients with hypercapnia } \\
\text { during spontaneous breathing trial }\end{array}$} \\
\hline & NIV & Conventional therapy & p-value \\
\hline Subjects $n$ & 25 & 22 & \\
\hline $\mathrm{Pa}, \mathrm{CO} 2 \mathrm{mmHg}$ & $55.6 \pm 6.6$ & $52.9 \pm 5.7$ & 0.19 \\
\hline Respiratory failur & $3(12)$ & $10(45)$ & 0.03 \\
\hline Reintubation & $3(12)$ & $8(36)$ & 0.10 \\
\hline $\begin{array}{l}\text { ICU length of sta } \\
\text { days }\end{array}$ & $14.5 \pm 9.8$ & $22.1 \pm 14.6$ & 0.04 \\
\hline Hospital mortality & $4(16)$ & $11(50)$ & 0.03 \\
\hline
\end{tabular}

averted potential worsening in respiratory status of OSA patients from residual effects of sedatives and narcotics that could have developed hours post-extubation.

Most of the published studies assessing the use of NIV in acute respiratory failure have relied on oro-nasal or facial masks [6, $7,18,19]$. Although there are no published studies, to the current authors' knowledge, on the superiority of one method over the other in the obese population, prior experience suggests a better tolerance for nasal masks in the morbidly obese, because these tend to be less claustrophobic. One controlled trial comparing the efficacy of nasal and oro-nasal masks in 26 nonobese patients with stable hypercapnia caused by chronic obstructive pulmonary disease (COPD) or restrictive disease found that the oro-nasal mask was more effective in lowering $\mathrm{Pa}, \mathrm{CO}_{2}$ but the nasal mask was better tolerated than either the nasal pillow or the oro-nasal mask [23]. However, another preliminary report from a controlled trial comparing nasal and oro-nasal masks found that the rates of decline of $\mathrm{Pa}_{\mathrm{a}} \mathrm{CO}_{2}$ and respiratory rate were independent of mask type when the masks were used for patients with respiratory distress [24]. Owing to the ongoing debate, further studies are needed to elucidate the optimal interface for the most effective response in obese patients with respiratory failure.

The results of the present study point to preferential benefit of NIV in severely obese patients with underlying hypercapnia. The effectiveness of NIV in reducing the rate of hypercapnic respiratory failure has been suspected from earlier studies involving patients with COPD exacerbations [25, 26]. In a nonrandomised trial, HILBERT et al. [26] reported 47\% attributable risk reduction in the need for endotracheal intubation for hypercapnic COPD patients assigned to NIV post-extubation. A significant decrease in mean duration of ventilatory assistance for the treatment of post-extubation distress and in the length of ICU stay was also noted. Comparable to the observations presented here, NIV was more effective in patients who exhibited hypercapnia during the spontaneous breathing trial. However, the present data extended those 
findings by demonstrating a reduced rate of bloodstream and hospital-acquired pulmonary infections, which were translated into shorter length of stay and improved hospital mortality. It is noteworthy that aside from possible random effects as a consequence of the limitations of post hoc analysis, the current study was not powered to address the outcome of prophylactic NIV in hypercapnic obese patients. This hypothesis should be investigated in a future prospective, randomised controlled trial.

Among the limitations of the present study is the use of historically matched controls [27]. A frequently cited source of bias with historical controls is the mere fact that implementing a prospective study may improve patient outcome. Moreover, historical comparisons of two cohorts are influenced by changes in disease patterns and treatment strategies, and are considered to favour the group treated with the new method. However, except for the application of NIV therapy, the presence of protocols-driven ICU management coupled with low staff turnover in the chosen institution would militate against this possibility. Moreover, differences in pulmonary function among participants might favour one group over the other, particularly if an obstructive pattern happens to be more frequent in the NIV group. In the absence of pulmonary function tests, this possibility should be taken into consideration when interpreting the results of the study. Another limitation of this study pertains to the potential of high prevalence of sleep apnoea in the study population, which might introduce a bias in favour of the NIV group. Pending a randomised controlled trial, the risk of respiratory complications and poor outcome of prolonged ventilation in this highrisk group population should be balanced against the observed benefits of a historical controlled investigation.

In conclusion, prophylactic use of noninvasive ventilation in severely obese patients post-extubation is warranted. Early application of noninvasive ventilation may be effective in averting respiratory failure before the development of respiratory distress and may be responsible for decreasing mortality in selected patients with chronic hypercapnia.

\section{ACKNOWLEDGEMENTS}

The authors are indebted to the respiratory therapist unit, the nursing staff of the intensive care units, and to the patients for their cooperation in the completion of this study.

\section{REFERENCES}

1 El Solh A, Sikka P, Bozkanat E, Jaafar W, Davies J. Morbid obesity in the medical ICU. Chest 2001; 120: 1989-1997.

2 Taylor RR, Kelly TM, Elliott CG, Jensen RL, Jones SB. Hypoxemia after gastric bypass surgery for morbid obesity. Arch Surg 1985; 120: 1298-1302.

3 Blouw E, Rudolph A, Narr B, Sarr M. The frequency of respiratory failure in patients with morbid obesity undergoing gastric bypass. AANA J 2003; 71: 45-50.

4 Gaszynski T, Gaszynski W, Strzelczyk J. Critical respiratory events in morbidly obese. Twoj Magazyn Medyczny Chirurgia 2003; 3: 55-58.

5 American Thoracic Society, European Respiratory Society, European Society of Intensive Care Medicine, and Societe de Reanimation de Langue Francçaise. International Consensus
Conferences in Intensive Care Medicine: noninvasive positive pressure ventilation in acute respiratory failure. $A m \mathrm{~J}$ Respir Crit Care Med 2001; 163: 283-291.

6 Esteban A, Frutos-Vivar F, Ferguson ND, et al. Noninvasive positive-pressure ventilation for respiratory failure after extubation. $N$ Engl J Med 2004; 350: 2452-2460.

7 Keenan SP, Powers C, McCormack DG, Block G. Noninvasive positive-pressure ventilation for postextubation respiratory distress: a randomized controlled trial. JAMA 2002; 287: 3238-3244.

8 Ferrer M, Valencia M, Bernardich O, et al. Non-invasive ventilation in the prevention of extubation failure. A randomized clinical trial. Am J Respir Crit Care Med 2004; 169: A523.

9 Bercault N, Boulain T, Kuteifan K, Wolf M, Runge I, Fleury J. Obesity-related excess mortality in an adult intensive care unit: a risk-adjusted matched cohort study. Crit Care Med 2004; 32: 998-1003.

10 Knaus WA, Draper EA, Wagner DP, Zimmerman J. APACHE II: a severity of disease classification system. Crit Care Med 1985; 13: 818-829.

11 MacIntyre N, Cook D, Wesley E, et al. Evidence-based guidelines for weaning and discontinuing ventilatory support: a collective task force facilitated by the American College of Chest Physicians, the American Association for Respiratory Care and the American College of Critical Care Medicine. Chest 2001; 120: Suppl. 6, 375S-395S.

12 Ely E, Baker A, Evans G, Haponik E. The prognostic significance of passing a daily screen of weaning parameters. Intensive Care Med 1999; 25: 581-587.

13 Joris J, Sottiaux T, Chiche J, Desaive C, Lamy M. Effect of bi-level positive airway pressure nasal ventilation on the postoperative pulmonary restrictive syndrome in obese patients undergoing gastroplasty. Chest 1997; 111: 665-670.

14 Ferrer M, Esquinas A, Arancibia F, Gonzalez G, Alarcon A, Torres A. Noninvasive ventilation during persistent weaning failure. A randomized controlled trial. Am J Respir Crit Care Med 2003; 168: 70-76.

15 Garner JS, Jarvis WR, Emori TG, Horan TC, Hughes JM. CDC definitions for nosocomial infections. I Infect Control 1988; 16: 128-140.

16 Epstein SK, Ciubotaru RL. Independent effects of etiology of failure and time of reintubation on outcome for patients failing extubation. Am J Respir Crit Care Med 1998; 158: 489-493.

17 Jiang J, Kao S, Wang S. Effect of early application of biphasic positive airway pressure on the outcome of extubation in ventilator weaning. Respirology 1999; 4: 161-165.

18 Nava S, Gregoretti C, Fanfulla F, et al. Noninvasive ventilation to prevent respiratory failure after extubation in high-risk patients. Crit Care Med 2005; 33: 2465-2470.

19 Ferrer M, Valencia M, Nicolas JM, Bernadich O, Badia J, Torres A. Early noninvasive ventilation averts extubation failure in patients at risk: a randomized trial. Am J Respir Crit Care Med 2006; 173: 164-170.

20 Erman MK, Loube DI. Perioperative complications in obstructive sleep apnea patients. Sleep Breathing 1997; 1: 3-10. 
21 Benumof JL. Obstructive sleep apnea in the adult obese patient: implications for airway management. J Clin Anesth 2001; 13: 144-156.

22 Gupta R, Parvizi J, Hanssen A, Gay P. Postoperative complications in patients with obstructive sleep apnea syndrome undergoing hip or knee replacement: a casecontrol study. Mayo Clin Proc 2001; 76: 897-905.

23 Navalesi P, Fanfulla F, Frigeiro P, Gregoretti C, Nava S. Physiologic evaluation of noninvasive mechanical ventilation delivered with three types of masks in patients with chronic hypercapnic respiratory failure. Chest 2000; 28: 1785-1790.

24 Kwok H, McCormack J, Cece R, Houtchens J, Hill N. Controlled trial of oronasal versus nasal mask ventilation in the treatment of acute respiratory failure. Crit Care Med 2003; 31: 468-473.

25 Plant PK, Owen JL, Elliott MW. Early use of non-invasive ventilation for acute exacerbations of chronic obstructive pulmonary disease on general respiratory wards: a multicentre randomised controlled trial. Lancet 2000; 355: 1931-1935.

26 Hilbert G, Gruson D, Portel L, Gbikpi-Benissan G, Cardinaud JP. Noninvasive pressure support ventilation in COPD patients with postextubation hypercapnic respiratory insufficiency. Eur Respir J 1998; 11: 1349-1353.

27 Sacks H, Chalmers T, Smith H. Randomized versus historical controls for clinical trials. Am J Med 1982; 72: 233-240. 